\title{
On the Translation of English Movie Titles
}

\author{
Lu Yin \\ Foreign Language Department \\ Hebei Polytechnic University \\ Tangshan 063000, Hebei, China \\ Tel: 86-315-229-8170 E-mail:wxx_1015@126.com
}

\begin{abstract}
The film, a popular art with both artistic and commercial values, is one of the most influential mass media. Film looks like a piece of mirror, which reflects all the respects of human society, including the material world and the spiritual world as well. With large quantities of English movies being introduced into China, more and more movie titles are translated into Chinese, some well done while some poorly done. Exploring the present situation of film titles, within the framework of audience-oriented approach, this paper tries to generalize some principles, such as faithfulness, cultural awareness, and combination of commercial and aesthetic effects, of film titles with abundant examples. Based on the foregoing analysis, some concrete techniques of film title translation are discussed, such as transliteration, literal translation and explication.
\end{abstract}

Keywords: Movie titles, Present situation, Principles, Techniques

\section{Introduction}

"Movie is so important that it has become the first arts of the human world," pointed out by French artist and novelist Mona (Baker, 2004, p.40). With the increase in cultural exchanges between China and the rest of the world, especially with the Western countries, the movie has gradually stood out as one of the important medium of communication in its own right.

Movie titles are always the first thing that the audiences come to know about new movies. Thus a right choice of movie title translation is of great importance to the successful release of a movie. A properly-translated English movie title should fulfill the following functions:

First: Providing information about the story for the audience by summarizing the main plot, revealing the theme, or offering some clue.

Second: Adding attraction to the movie and stimulating the audience's interest in and desire for viewing the film.

Third: Saving trouble for the cinema, the audience, film reviewers and other research workers in their publicity, choice of viewing, comments and studies.

Like many other forms of non-literary translation, the translation of English movie titles has not yet received due attention despite their importance. Peter Newmark has called on the translators to bring their attention to "two underplayed aspects of translation", one of which is "the approach to non-literary translation." (2001, p.135).Nida also called for "more attention to exploring new fields in translation" (2004, p.17). This paper, based on the analysis of the present situation, will introduce some principles and techniques of English movie title translation.

\section{Techniques of English movie title translation}

Techniques of English movie title translation mainly fall into two categories: showing respect for the original title (transliteration, literal translation, explication) and discarding the original one (adaptation, providing a new title). "No matter what technique is employed, one fundamental and vital principle that should never be forgotten is that the translation must be related to the story in one way or another."( Eugene A,2001,p.214).

\subsection{Transliteration}

As English movies often have their settings, heroes' or heroines' names as titles, transliteration, i.e. borrowing the sound of the SL, can be employed when titles are place or personal names, especially when these names are familiar to the target audience---Chicago ( $<<$ zhi jia ge $>>)$, Casablanca $(<<\mathrm{ka}$ sa bu lan ka $>>)$ or of historic or cultural importance---Gandhi ( $<<$ gan di zhuan $>>)$, Aladdin $(<<$ a la ding $>>)$. Through transliteration, the sound effects, i.e. the 
rhythm of the SL, can be preserved, which will entertain the audience with a strong exotic flavor, such as Titanic $(<<$ tai tan ni ke $>>)$, Oliver (<<ao li fu) $>>$ and Nodding Hill $(<<$ nuo ding shan $>>)$.

\subsection{Literal translation}

By converting the SL grammatical constructions to their nearest TL equivalents, literal translation can preserve both the content and the form of the original to a maximum. On occasions when a title matches the story of a movie perfectly---Driving Miss Daisy, Saving Private Ryan, Around the World in 80 Days, and if the TL equivalents--- $<<$ wei dai xi xiao jie kai che $>>,<<$ zheng jiu da bing rui en $>>,<<$ huan you shi jie 80 tian $>>$, not sounding awkward or unnatural, are capable of describing the story, literal translation can be adopted. As most English movie titles consist of nouns or noun phrases, literal translation is the simplest and most effective technique used in movie title translation. Most descriptive titles, such as Roman Holiday ( $<<$ luo ma jia ri $>>)$, Gladiator $(<<$ jiao dou shi $>>)$, Man in Black $(<<$ hei yi ren $>>$ ) and Dances with Wolves ( $<<$ yu lang gong wu $>>)$, may find this technique applicable. And so are a small part of allusive ones like Scent of a Woman $(<<$ nv ren xiang $>>)$, Brave Heart $(<<$ yong gan de xin $>>)$.

\subsection{Explication}

To some titles, especially some allusive ones, whose literal translation cannot describe the story neatly and thus fails to provide information and attract the audience, more details have to be added to make them more explicit. Instead of being chosen randomly, these details should be based on the story and able to help polish the titles.

Example 4-1

Bambi ( $<<$ xiao lu ban bi $>>)$ and Shrek ( $<<$ guai wu shi lai ke $>>)$ are more lovable than $<<$ ban bi $>>$ and $<<$ shi lai ke $>>$. xiao lu and guai wu bring out the loveliness of the characters.

Example 4-2

In High Ice ( $<<$ bing feng qiang xian dui $>>)$ and Speed ( $<<$ sheng si shi su $>>)$, both qiang xian dui and sheng si highlight the tension of the story.

Example 4-3

If Seven is rendered simply into qi1, the audience will surely be bewildered. But if specified as qi zong zui (lust yin yu, vanity jiao ao, wrath fen nu, sloth lan duo, envy ji du, gluttony bao shi and avarice tan luan), the movie will be easily classified as a detective one.

Example 4-4

Nikita ( $<<$ nv qiu ni ji ta $>>)$ and Scarface $(<<$ ba lian da dao $>>)$ reveal the identities of our heroine and hero through the added information nv3qiu2 and da4dao4. Then the audience will wonder what has happened or will happen to her or him?

Example 4-5

JFK $(<<$ ci sha ken ni di $>>)$ and Ghost $(<<$ ren gui qing wei liao $>>)$ further specify the main plot of the story by explicating the two titles through ci sha and ren gui qing wei liao

Example 4-6

In the translation of Water World ( $<<$ wei lai shui shi jie $>>)$, wei lai tells the audience that it is a sci-fi movie, so it is no wonder that the world is full of water.

Example 4-7

The title, Piano, offers a clue to the story. And either gang qin bie lan or gang qing ke is more vivid and appealing than its literal translation gang qin. The Taiwan version goes even further---gang qin shi he ta de qing ren.

\subsection{Adaptation}

Adaptation is to change the cultural references. Bearing in mind the principle of cultural awareness, the translator must first understand the cultural information within movie titles, and then exert himself to find proper Chinese equivalents, which can be understood, accepted and appreciated by the Chinese audience easily.

Example 4-8 Salt of the Earth

The original is an allusion in the Gospel According to Matthew, referring to any person or persons regarded as the finest, noblest. This metaphor could not be well received by the Chinese audience if the title is literally rendered into $<<$ di qiu de yan $>>$. Instead, $<<$ she hui zhong jian $>>$ can be better understood by the Chinese audience.

Example 4-9 Chickpeas

A group of Beirut immigrants strive to start an undertaking in America. However, under the cultural impact, they are caught in a dilemma, just like the peas squeezed in the pods, not knowing whether to accept the American way of life or not. But this could not be effectively expressed by Chickpeas' literal translation $<<$ ying zui dou $>>$. The translator has done a good job in discarding the original, and choosing a concept more familiar to the Chinese audience--- $<<$ mei guo meng $>>$, through which the audience can easily understand the theme of the movie.

Example 4-10 One Flew over the Cuckoo's Nest

The Taiwan rendering of this title is $<<$ fei yue du juan wo $>>$, the literal translation of the Cuckoo's Nest. In fact, in English "cuckoo" is also a slang word, meaning a foolish or crazy person, for cuckoos always lay their eggs in the nests 
of other birds. And the Cuckoo's Nest refers to the madhouse. So it is proper to render it into $<<$ fei yue feng ren yuan $>>$. 2.5 Providing a new title

Language in movie titles is creatively used. Certainly there are titles to which the four techniques above are not applicable. Thus the translator sometimes has to provide a new title for the movie and rely on his own intuition and taste to choose one good translation among all the possible translations. The new title should factually and accurately describe the story.

Example 4-11 We Were Soldiers

The movie is adapted from the memoir of a veteran who once served in the Vietnam War and suffered much from it. The rendering <<zheng zhan sui yue $>>$ exactly expresses the past tense in the original--- "Were" reveals the identity of the heroes, and "Soldiers" more importantly and appropriately conveys a mixed feeling for that war.

Example 4-12 Forrest Gump

If it were simply transliterated into $<<$ fu rui si te-gang pu $>>$, surely, a good many audience would not have liked the movie when they first come to know the title. But $<<$ a gan zheng zhuan $>>$, a parody inspired by Lu Xun's $<<$ a Q zheng zhuan $>>$, which is rather well-known in China, sounds more appealing and will immediately arouse the audience's desire to watch the movie.

Example 4-13 The Professional

The title refers to the hero of the movie, Leon, a killer, very skillful and cool. One of its rendering is $<<$ sha shou lai ang $>>$. Appropriate to the identity of our hero as it is, it does not reveal another important side of Leon, which the rendering <<zhe ge sha shou bu tai leng $>>$ vividly carries---his tenderness and affection towards our heroine, a little orphan girl.

Example 4-14 Rain Man

The mainland rendering is $<<y \mathrm{yu}$ ren $>>$, which makes no sense to the Chinese audience, for in Chinese there is no such thing as yu ren. The movie is named so because one of our heroes in the movie, the older brother Raymond Babbitt, an autistic, always fails to pronounce his own name Raymond right, but makes a sound of Rain Man. The HK rendering $<<$ shou zu qing wei liao $>>$ is more suitable to the story.

\section{Conclusion}

In short, the translation of film titles involves many complicated factors. It depends on the content of films, the psychology of the audience, and some other cultural factors. Anyway, it must be a good version if it is concise, striking, attracting and meaningful. As we have analyzed above, a good rendering of film titles should obey such principles: faithfulness, cultural awareness, combination of commercial and aesthetic effects. Transliteration, literal translation and explication are the usual methods we use when we translate film titles. Only by bearing in mind the principles of faithfulness, cultural awareness, and combination of commercial and aesthetic effects, and employing techniques appropriate to different titles, can we translators do a better job when translating film titles.

\section{References}

Baker, Mona. (2004). Routledge Encyclopedia of Translation Studies. Shanghai: Shanghai Foreign Language Education Press.

Cameron, Lynne \& Graham Low. (2001). Researching and Appling Metaphor. Shanghai: Shanghai Foreign Language Education Press1.

Eugene A. Nida. (2001). Language and Culture: Contexts in Translation. Shanghai: Shanghai Foreign Language Education Press.

Gentzler, Edwin. (2004). Contemporary Translation Theories. Shanghai: Shanghai Foreign Language Education Press.

Hickey, Leo. (2001). The Pragmatics of Translation. Shanghai: Shanghai Foreign Language Education Press.

Kelly, Jeanne \& Nathan K. Mao. (2003). Fortress Besieged. Beijing: Foreign Language Teaching and Research Press.

Lakoff, G \& M. Johnson. (1980). Metaphors We Live By. Chicago and London: The University of Chicago Press.

Newmark, Peter. (2001). A Textbook of Translation. Shanghai: Shanghai Foreign Language Education Press.

Nida, Eugene A. (2004). Toward a Science of Translating. Shanghai: Shanghai Foreign Language Education Press.

Snell-hornby, Mary. (2001). Translation Studies: An Integrated Approach. Shanghai: Shanghai Foreign Language Education Press. 